\title{
Derivação ventriculoatrial percutânea - Nota técnica
}

\author{
Adriano Donizeth Silva de Morais ${ }^{1}$, Mayana Menegusse Mesquita ${ }^{1}$, Thiago da Silva \\ Santos ${ }^{1}$, Cícero Anderson da Cruz Macedo Tavares ${ }^{1}$, Leonardo Miranda de Avellar ${ }^{2}$ \\ Serviço de Neurocirurgia do Hospital Geral Roberto Santos, Salvador, BA, Brasil.
}

\section{RESUMO}

Descrição passo a passo de técnica de derivação ventriculoatrial utilizando punção cervical percutânea por técnica de Seldinger. O objetivo é demonstrar e difundir entre os neurocirurgiões brasileiros uma forma alternativa segura, rápida e eficaz de introdução e instalação de cateter venoso até o átrio direito, sem necessidade de dissecção venosa convencional. São vantagens a redução do tempo cirúrgico e o melhor resultado cosmético, sem aumento significativo do custo.

\section{PALAVRAS-CHAVE}

Hidrocefalia/complicações, derivações do líquido cefalorraquidiano, infecção, hipertensão intracraniana, ventriculite cerebral.

\section{ABSTRACT}

Percutaneous ventriculoatrial shunt - Technical note

Step-by-step technique using ventriculoatrial shunt through percutaneous cervical puncture by Seldinger's technique. The goal is to demonstrate and spread among Brazilian neurosurgeons an alternative safe, fast and effective way to introduce and install venous catheter up to the right atrium without the need for conventional venous dissection. Advantages of the method are shorter surgical time and better cosmetic results, without significant increase in the cost.

\section{KEYWORDS}

Hydrocephalus/complications, cerebrospinal fluid shunts, infection, intracranial hypertension, cerebral ventriculitis.

\section{Introdução}

A via vascular é um importante método para as derivações ventriculares no tratamento de hidrocefalia, todavia seu maior potencial de complicação e o advento e aperfeiçoamento da via peritoneal reservaram a via venosa a casos restritos, tanto em adultos quanto em crianças. ${ }^{1-3}$ Dissecção venosa cervical foi o método inicialmente descrito para inserção do cateter distal, e dificuldades anatômicas podem aumentar o tempo cirúrgico por esse método. ${ }^{3}$ Este trabalho descreve um método de inserção do cateter distal de derivação ventriculoatrial (DVA) por punção venosa cervical direta, sem necessidade de dissecção.

\section{Materiais e métodos}

\section{Técnica cirúrgica}

Descrevemos um procedimento de DVA em uma paciente de 13 anos, com diagnóstico de hidrocefalia e múltiplas tentativas malsucedidas de derivação ventriculoperitoneal prévias, evoluindo com extensas cicatrizes de parede abdominal e aderências peritônio-intestinais.

Optou-se por iniciar com a punção venosa, com o objetivo de reduzir o tempo de exposição da dura-máter. Estudo venoso com USG (ultrassonografia) Doppler cervical pode ser feito nos casos em que múltiplas punções

1 Médicos-residentes do Serviço de Neurocirurgia do Hospital Geral Roberto Santos, Salvador, BA, Brasil.

2 Neurocirurgião chefe de equipe e coordenador da Residência do Serviço de Neurocirurgia do Hospital Geral Roberto Santos, Salvador, BA, Brasil. 
anteriores tenham sido realizadas no lado acessado. Fez-se a punção da veia jugular pela técnica de Seldinger convencional, com cateter venoso duplo lúmen $6 \mathrm{Fr}$, e introdução da agulha no ápice do triângulo formado pelas cabeças clavicular e esternal do músculo esternoclidomastóideo, com direção ao mamilo ipsilateral (Figura 1). A veia subclávia também pode ser usada por punção-padrão.

Após a punção, foi introduzido o arame-guia, orientado pela fluoroscopia, até o átrio direito, na altura dos níveis vertebrais T4 a T7. Retirou-se a agulha de punção, com adaptações subsequentes do introdutor e do dilatador do sistema de cateter vascular 6Fr, que apresenta calibre ideal para passagem do cateter atrial (Figura 2). A passagem deste deu-se após a retirada do introdutor. Utilizamos DVP Codman ${ }^{\circledast}$, pois apresentou o diâmetro de cateter ideal para passagem pelo introdutor (Figura 3).

O procedimento de cateterização ventricular seguiu a técnica-padrão, com trepanação em ponto de Dandy à direita. O lado direito também foi o escolhido por causa da orientação menos sinuosa da veia cava interna desse lado, facilitando sua punção. Fez-se, então, a tunelização do cateter distal até o ponto de punção venosa (Figura 4).

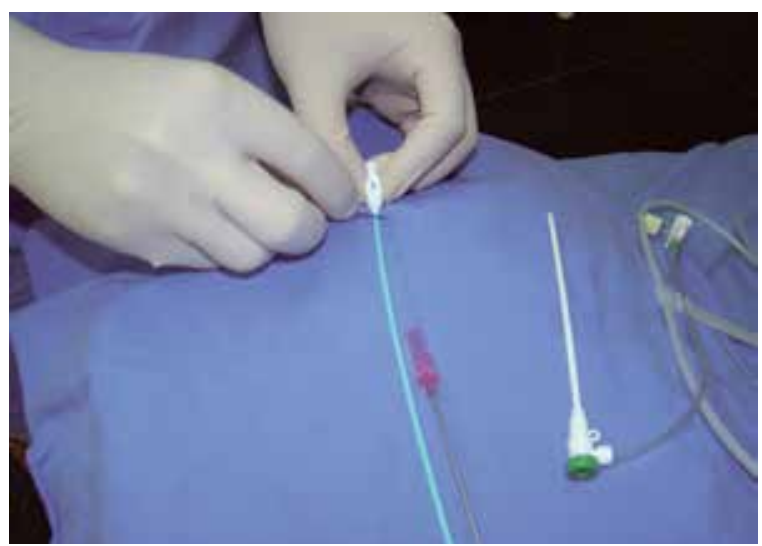

Figura 1 - Cateter 6Fr (agulha de punção, fio-guia metálico, dilatador e introdutor) sendo adaptado para passagem do cateter atrial.

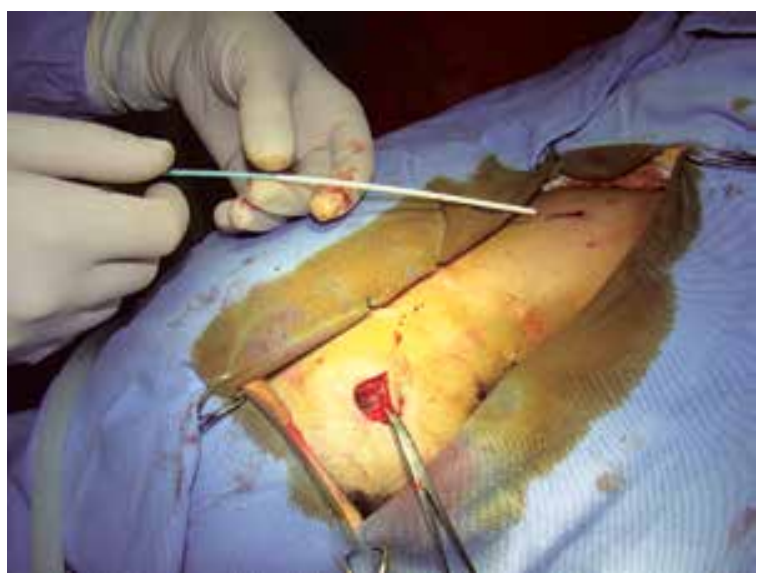

Figura 2 - Passagem do introdutor. Incisão cefálica, sem trepanação.

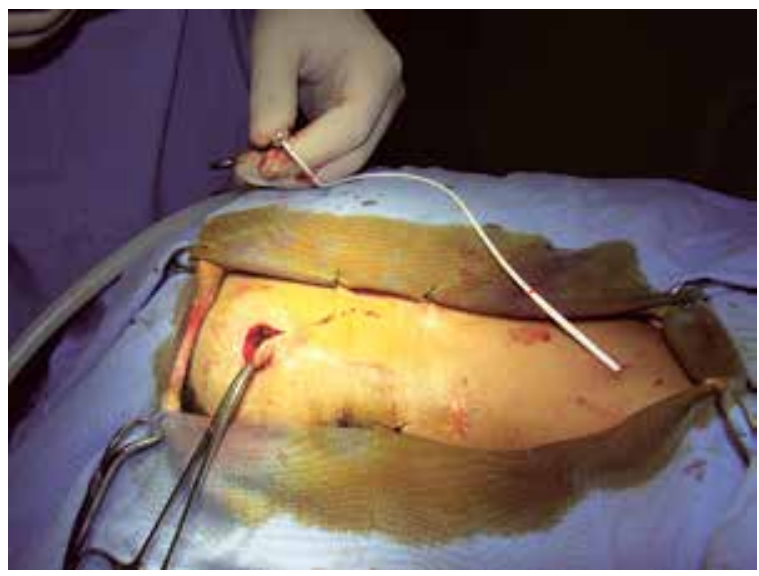

Figura 3 - Retirada do introdutor e passagem percutânea do cateter ventricular; nesta etapa é feita a trepanação.

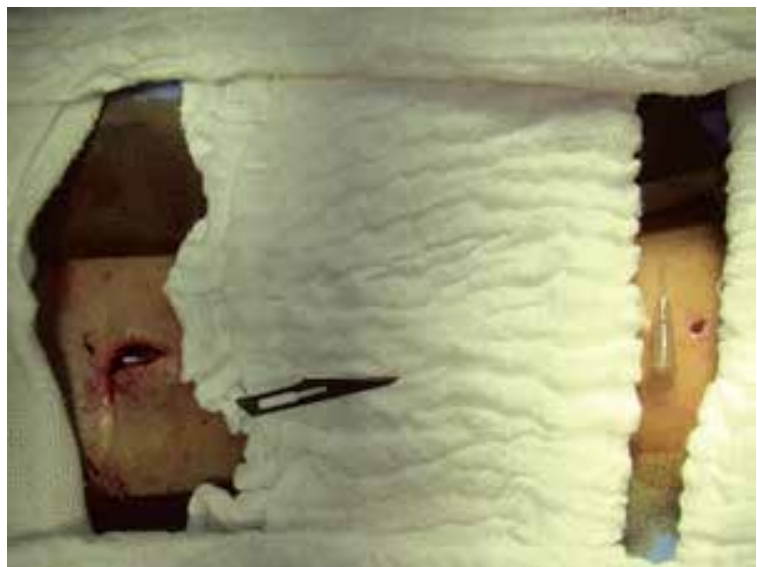

Figura 4 - Incisão cervical puntiforme após instalação de todo o sistema.

O seguimento ambulatorial do caso é feito há dois anos, sem complicações até o momento. Tomografias de crânio posteriores demonstram cateter ventricular bem posicionado. A paciente não apresenta sintomas ou sinais clínicos de problemas cardiovasculares de qualquer natureza.

\section{Resultados}

O follow-up de dois anos revela bom funcionamento do sistema valvular, sem complicações aparentes. Houve recuperação do padrão neurológico, sem déficits adicionais. Controles tomográficos e radiológicos mostram bom posicionamento dos cateteres cefálico e atrial, com hidrocefalia compensada. 


\section{Discussão}

Apesar de há mais de duas décadas já haver relatos de casos com notas técnicas descrevendo o método percutâneo para introdução do cateter atrial nas derivações para tratamento de hidrocefalia, essa técnica é ainda pouco conhecida e subutilizada em hospitais brasileiros. ${ }^{4} \mathrm{~A}$ literatura ressalta as vantagens observadas em nosso caso, como redução de tempo cirúrgico (e seus benefícios), melhor resultado cosmético e ausência de custo adicional relevante. ${ }^{5-7}$

No decorrer do procedimento, optamos primeiramente pela punção venosa, pois, uma vez que haja dificuldade nela e prolongamento do ato cirúrgico, mantemos menor tempo de exposição do sistema nervoso central e da válvula ao ar ambiente. Não realizamos incisão no ponto de punção cervical, já que a abertura de pele produzida pelo dilatador é suficiente para passagem do cateter atrial.

Enfatizamos que situações de contraindicação de DVP necessitam de uma via alternativa segura e eficaz para derivações ventriculares permanentes. Ao longo destas últimas décadas, viu-se que as complicações específicas relacionadas ao uso do cateter não são muito mais prevalentes que aquelas da DVP, mas que tendem a ser mais toleradas que as de vias menos frequentes, como o cateter distal pleural ou outros.

Novos relatos e estudos seriados devem apontar qual a melhor forma de identificar precocemente tais complicações e a melhor conduta a ser adotada. Trombose venosa e infecção, como meningite (mais precoce) e endocardite (mais tardia), ainda parecem ser as mais importantes, mas há também relatos de defeitos de válvula tricúspide, calcificação e perfuração da parede cardíaca, decorrentes do contato direto do cateter com o miocárdio, além de complicações associadas à técnica de punção venosa, como o pneumotórax. ${ }^{1,2}$

\section{Conclusão}

A técnica de punção percutânea para DVA mostra-se segura e eficaz e traz vantagens adicionais, como menor tempo de procedimento e menor trauma cirúrgico, já que prescinde de dissecção venosa. O custo e os efeitos colaterais não foram elevados significativamente.

\section{Referências}

1. Engelman RM, Ransohoff J, Cortes LE, Spencer FC. Complications of ventriculoatrial shunting for hydrocephalus requiring cardiac operation. Ann Thorac Surg. 1969;8(5):464-9.

2. Mujanovic E, Bergsland J, Jurcic S, Avdic S, StanimirovicMujanovic S, Kabil E. Calcified right atrial and pulmonary artery mass after ventriculoatrial shunt insertion. Med Arh. 2011;65(6):363-4.

3. Gonzalez LF, Kim L, Rekate HL, McDougall CG, Albuquerque FC. Endovascular placement of a ventriculoatrial shunt. Technical note. J Neurosurg. 2007;106(Suppl 4):319-21.

4. Ashker K, Fox JL. Percutaneous technique for insertion of an atrial catheter for CSF shunting. Technical note. J Neurosurg. 1981;55(3):488-90.

5. Chuang $\mathrm{HL}$, Chang $\mathrm{CN}$, Hsu JC. Minimally invasive procedure for ventriculoatrial shunt-combining a percutaneous approach with real-time transesophageal echocardiogram monitoring: report of six cases. Chang Gung Med J. 2002;25(1):62-6.

6. Harrison MJ, Welling BG, DuBois JJ. A new method for inserting the atrial end of a ventriculoatrial shunt. Technical note. J Neurosurg. 1996;84(4):705-7.

7. Kim YJ. Percutaneous insertion of the distal catheter during ventriculo-atrial shunts. A simple and reliable method. J Korean Neurosurg Soc. 2007;41:429-31.

Endereço para correspondência

Adriano Donizeth Silva de Morais

Rua Desembargador Manoel Pereira, 55,

ap. 205, Bairro Costa Azul

41760-250 - Salvador, BA, Brasil

E-mail: adrianomorais83@gmail.com 